sides of the Atlantic which suggest with increasing certainty that a phase transition occurs in liquid ${ }^{3} \mathrm{He}$ near $0.002 \mathrm{~K}$. This transition is closely analogous to the superconducting transition in a metal.

According to quantum statistical mechanics, the properties of a fluid at very low temperatures are determined chiefly by whether the atoms of which it is composed contain an even number of fundamental particles, in which case they are bosons, or an odd number, in which case they are fermions. The ${ }^{4} \mathrm{He}$ atom ( 2 protons, 2 neutrons, 2 electrons) is thus an example of a boson and the ${ }^{3} \mathrm{He}$ atom, with one less neutron in the nucleus, is a fermion. The two liquids are therefore expected to display completely different properties at very low temperatures, a prediction which was verified experimentally several years ago when ${ }^{3} \mathrm{He}$ first became available in quantities sufficient for experiments. Superfluidity in liquid ${ }^{4} \mathrm{He}$ is associated with a curious phenomenon, known as Bose-Einstein condensation, in which a substantial proportion of the atoms congregate in the same zero-energy quantum state. By contrast, the occupation of a single quantum state by more than one fermion is rigorously forbidden, so that a Bose-Einstein condensation, leading to superfluidity, cannot occur in liquid ${ }^{3} \mathrm{He}$.

There is, however, a completely different mechanism by which the electrons (also fermions) in a superconductor are able to acquire their superfluid properties. This is the formation of so-called Cooper pairs: where there is an attractive force between the electrons, on account of their interaction with the lattice of positive ions, the assembly can reduce its total energy by forming pairs of electrons. The scattering of electrons by lattice defects or thermal vibrations, which gives rise to electrical resistance in a normal metal, would entail breaking pairs and thus cannot occur unless a certain minimum amount of energy is available. The electrons can therefore flow through the lattice without dissipation of energy, provided that a critical drift velocity is not exceeded. In the case of liquid ${ }^{3} \mathrm{He}$, the possibility of attractive interactions, which might enable a similar transition to the paired state to occur, was considered more than a decade ago by several workers who estimated transition temperatures around $0.1 \mathrm{~K}$. Subsequent experiments failed to reveal a transition; but more refined theories quickly pushed the predicted transition to still lower temperatures. This cyclical process has been repeated several times since then, but on each occasion the transition has failed to appear at the temperature expected.

The whole topic attracted renewed interest last year when Osheroff, Gully, Richardson and Lee (Phys. Rev. Lett., 29, 920 ; 1972) reported evidence of some sort of phase transition in liquid ${ }^{3} \mathrm{He}$ under high pressure near $0.002 \mathrm{~K}$. Unfortunately, however, their data were hard to interpret in detail because their cooling technique meant that, inevitably, some solid ${ }^{3} \mathrm{He}$ was also present in the experimental cell.

The experiments by Webb et al. made use of a different cooling method, and provide the first clear indication that the phenomenon observed by Osheroff et al. is indeed the long-awaited transition to a paired state: they find that no latent heat is associated with the transition, but that there is a finite discontinuity in the specific heat. This behaviour is characteristic of a secondorder phase transition, the only other known example of which is the superconducting transition in a metal.

Does the new phase of liquid ${ }^{3} \mathrm{He}$ have superfluid properties? Observation of the second-order phase transition leaves little doubt that there are close similarities between this new phase and the electrons in a superconductor, but clearly there are also profound differences. In a superconductor the fermions are negatively

\title{
Integrating Two Unrelated DNA Sequences
}

Mosr bacteriophage DNAs which can be inserted into the chromosome of a bacterial host on infection can integrate at only one, or at least a very few, sites in the bacterial genome. A striking exception is provided by phage mu, which can integrate at any point in the chromosome of Escherichia coli. When it inserts within a gene, the bacterium behaves as mutant lacking the gene function. This unusual activity has already been put to practical use by Nomura and Edbaek (Proc. US Nat. Acad. Sci., 69, 1526 ; 1972) to map the relationship of the genes coding for ribosomal proteins and by Louarn, Bird and Caro (J. Mol. Biol., 70, 549 ; 1972) to demonstrate the bidirectional replication of the $E$. coli chromosome.

The mechanism of this integration is analysed in an article by Toussaint and Faelen in Nature New Biology next Wednesday (March 7). They have taken advantage of their previous observation that phage mu can promote the integration in the bacterial chromosome of a defective lambda phage (which usually inserts at one specific site) unable to integrate of its own accord. If the lambda phage carries an active $\mathrm{gal}^{+}$gene, its integration is marked by the restoration to infected cells (of the $\mathrm{gal}^{-}$genotype) of ability to metabolize galactose as a carbon source.

The question which Toussaint and Faelen answer is whether mu promotes the integration of lambda simply by providing some enzymatic activity or whether the two DNAs are physically linked in some way so that lambda is inserted at the same site as mu. They have therefore isolated cells in which $\lambda \mathrm{gal}^{+}$has been inserted within a bacterial gene (by screening for the inability of the cell to provide the metabolic activity coded by that gene). The next step in the analysis is to see which of the integrated phages is lost if the bacterial gene bearing these insertions is replaced by a normal, functional bacterial gene. This experiment is per- formed by transducing the cells with a P1 phage.

In a his ${ }^{-} E$. coli strain which had received $\mathrm{Agal}^{+}$and $\mathrm{mu}$, transduction to provide a normal $\mathrm{his}^{+}$bacterial gene resulted in the loss of both $\lambda \mathrm{gal}$ and $\mathrm{mu}$. This implies that both phage DNAs must have integrated at the same site, within the his gene. In further experiments, the chromosomes of cells bearing $\mathrm{Agal}^{+}$were transferred by conjugation into $\mathrm{gal}^{-}$bacteria. All the $\mathrm{gal}^{+}$ recipients received both the lambda and mu DNAs, which supports the idea that $\mathrm{mu}$ enables lambda to integrate because the two phages are linked together in some way. In these different strains, the $\mathrm{gal}^{+}$was located at widely differing points in the donor chromosome, showing that mu retained its usual capacity to integrate at any point, the integration of $\lambda \mathrm{gal}$ taking place at the same site.

The model which Toussaint and Faelen propose to explain these results is to suppose that two phage mu DNAs are implicated in the integration process. The two mu DNAs interact with each other at the specific sites which the phage uses for integration to form a dimer. One of the two sites of this dimer integrates in gal DNA ; the other integrates in the bacterial chromosome. The result is that the bacterial insertion site carries a $\lambda$ gal genome, surrounded on each side by a mu genome. Studies of the structure of the DNA which is inserted support this model. The use of mutants in phage mu shows that the same phage functions are implicated in its interaction with $\lambda g a l$ and with bacterial DNA

One implication of this model is that in principle it should be possible to utilize mu to connect together any two circular pieces of duplex DNA in the bacterial cell. Although this model is consistent with the genetic data, biochemical confirmation of the proposed DNA interactions will be needed before it can be put to such interesting and important uses. 